\title{
Study on Application of Infrared Spectrum Technology in Power Equipment
}

\author{
Yongxiang $\mathrm{Li}^{1}$, Tianzheng Wang ${ }^{1}$, Hui Yuan ${ }^{1}$, Gang Yang ${ }^{1}$ and Na Zhang ${ }^{1}$ \\ ${ }^{1}$ Shanxi Electric Power Research Institute, Taiyuan, China
}

\begin{abstract}
In this paper, the application of infrared spectrum acquisition technology in power equipment is studied. Infrared spectrum identification and analysis technology and fault diagnosis technology can monitor and analyze the operation parameters of important equipment in real time, so as to improve the work efficiency of substation staff and achieve higher economic benefits. Therefore, infrared spectrum technology has great practical value and application prospect in power equipment.
\end{abstract}

\section{Introduction}

With the rapid development of China's economy, a large number of accesses of the UHV AC / DC hybrid and new energy have became the inevitable trend of development of power grid, and the present stage of China's power grid covering a wide range of power equipment in large quantities, so new demands on the unified management and monitoring and operation management of power equipment have been put forward[1]. At present, the video surveillance system can realize the functions of field equipment monitoring, remote control camera movement, digital video recording and other functions, but it can not realize image identification function. So the development of image identification function becomes the most important problem. Infrared spectrum technology can make up for this deficiency, so the research of this technology is imperative[2].

\section{Theoretical technology}

\subsection{Infrared spectrum identification and analysis technology}

The infrared spectrum of power equipment mainly reflects the distribution of temperature, so it does not show the details of equipment characteristics. Therefore, the identification of infrared spectrum of power equipment needs to identify and mark the equipment through visible light, and establish two-way equipment type tagging model.

Among them, the visible light on the surface of the equipment marked text and signs: We design and study the core algorithm for optical character identification system which is suitable for most machine vision fields, and annotate the image by using the identification results. Then, the feasibility and practicability of the algorithm are tested, and the algorithm with strong anti noise ability and good effect is selected. The core algorithms required for optical character identification systems include the following aspects[3]:

First, image preprocessing steps: Power equipment image preprocessing is necessary, including enhancing the quality of the input image, image denoising, edge enhancement, edge detection, etc. The digital image is located and cut out the region of interest through the image processing algorithm. On the basis of the region of interest, we continue to segment and extract the related structure according to the specific task requirements. We need to collect and ensure the accuracy of the electrical equipment and its operation state identification and analysis;

Second, feature extraction step: The key to extract feature vectors from discrete feature digital images is to extract feature vectors with high difference between feature;

Third, pattern identification step: We input the extracted feature vectors, and identify and describe them by pattern matching algorithm, and then divide the features correctly and complete the task of image processing;

Fourth, the image is annotated with the identification result.

As for the image without text sign in image area: This paper studied multi-scale spatial model and establishes a simple system of image pyramid, which is simple and effective to explain multi-scale image features. It is convenient to add scale space information in image feature extraction; the research combines the traditional supervised classifier with deep learning, which makes the algorithm perform well in the condition of large data and small data. In the implementation process, the depth learning method is usually selected to extract the sample 
features, and the traditional supervised classifier method is used to classify the samples[4].

For the automatic annotation of the image of this kind of equipment, firstly, the image processing technology is used to extract the underlying visual features of the equipment image, including color, texture, shape and spatial information, which are used as the metadata of the image. For the image annotation of a power equipment, the annotation problem is considered as an image classification problem, which is divided into two stages:

First, annotation model training stage (using a large number of classified images to train classifiers): We need to submit images representing the specific visual requirements of the project, and use the annotated image set to construct a deep network mapping model from the underlying visual features of the image to the high-level semantic features abstracted layer by layer.

Second, image annotation stage: we will calculate the similarity of all the images in the training library and return the most similar image to it. According to the visual information of the test image, it is classified into predefined categories. Each keyword is considered as an independent category name and corresponds to a classifier. Thus, we can more accurately mark the unknown samples of power equipment image. The internal frame diagram of the whole design system is described in figure[5].

\subsection{Study on infrared spectrum fault diagnosis technology}

At present, the infrared detection device has the function of automatically judging the temperature, but it is only limited to the point in the fixed angle picture, and the equipment may have a variety of fault points, and the situation is complex. And this point belongs to the equipment still needs manual judgment. Therefore, as for the equipment fault diagnosis using infrared spectrum, the infrared spectrum should be preprocessed firstly, then the temperature field segmentation based on the isotherm is carried out to determine the defect center position of the infrared spectrum. Then, the visible image is identified and matched, so that the fault diagnosis is carried out. The flowchart can be represented by the following block diagram(figure 1):

\subsubsection{Study on preprocessing of infrared spectrum}

Infrared thermal spectrum has the following characteristics:

a. Because of the thermal balance of the scene, the wavelength of light wave, the distance of transmission, the atmospheric attenuation and other reasons, the infrared image has strong spatial correlation, low contrast and blurred visual effect;

b. Infrared thermal images show that the temperature distribution of the scene is gray image without color or shadow, so it has low resolution and poor resolution potential for human eyes;

c. The detection capability and spatial resolution of the thermal imaging system are lower than the visible CCD array, which makes the infrared image less clear than the visible image;

d. The random noise of external environment and the imperfection of thermal imaging system bring a variety of noise to infrared image, such as thermal noise, shot noise, photon electronic fluctuation noise and so on.

e. Due to the inconsistency of the response characteristics of the infrared detector and the defects of the optical scanning system, the non-uniformity of the infrared image is caused by the fixed pattern noise, reflecting crosstalk, distortion and so on.

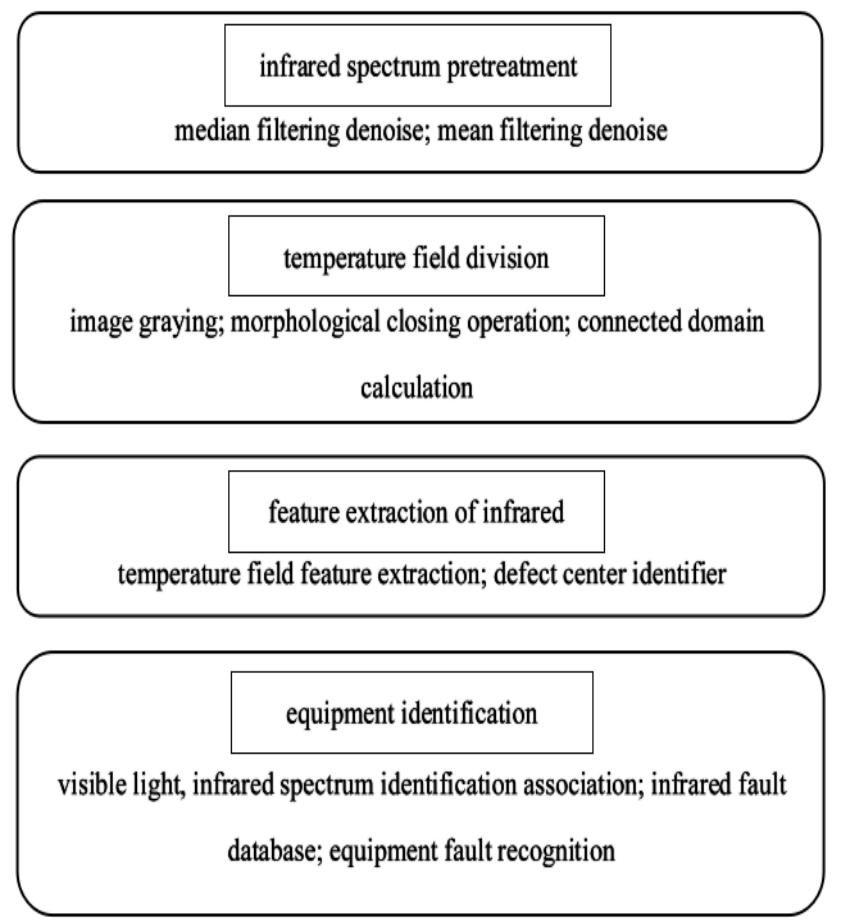

Figure 1: flowchart of external graph fault diagnosis

From the above analysis, we can see that the infrared image is generally dark, and the contrast between the target image and the background is low, the edge is fuzzy, and the noise is large.

According to these characteristics of infrared spectrum, the mean filtering and median filtering algorithm are needed to achieve the purpose of smoothing the noise of infrared image preprocessing[6].

Mean Filter. Image to the target pixel, which includes the adjacent pixels and its own pixels. Replace the original pixel value with the average of all pixels in the template. Mean filter is also called linear filtering, and its main method is neighborhood averaging method. The basic principle of linear filtering is to replace each pixel value in the original image with the mean value, which means that the current pixel $(\mathrm{x}, \mathrm{y})$ is treated. You need to select a template that consists of several pixels adjacent to it. It will find the mean of all pixels in the template. Then, the mean value is given to the current pixel $(\mathrm{x}, \mathrm{y})$ as the gray value $\mathrm{g}(\mathrm{x}, \mathrm{y})$ of the image after processing, that is, $g(x, y)=1 / \mathrm{m}$ sigma $\sum f(x, y)$, and $M$ is the total number of pixels in the template, including the current pixel. The mean filter can effectively filter the additive noise in the image, but the mean filter itself has inherent defects, and it cannot well protect the image details in the 
image denoising and destroys the image details, so that the image becomes blurred. Mean filter are arithmetic mean filtering, geometric mean filtering, harmonic filtering and inverse harmonic mean filtering. This project aims to verify the noise reduction effect on arithmetic mean filter, geometric mean filter and inverse.

Median Filter. Median filter is a commonly used nonlinear smoothing filter. The basic principle is to use the median value to replace the value of a neighborhood of the point in a digital image or sequence. The main function is to make the pixels with different gray values of the surrounding pixels change the value close to the surrounding pixel value, so as to eliminate the isolated noise points. So median filtering is very effective for filter the salt and pepper noise. The conventional median filter has a good smoothing effect on the noise of long tailed probability distribution[7]. It not only eliminates noise, but also equips the advantage of protecting boundary information, and plays a protective role in some details of the image, so it has been widely used in image denoising processing. However, the performance of conventional median filter de impulse noise is greatly affected by the size of filter window. And it has some contradictions in suppressing image noise and protecting details in two aspects: The smaller the filter window is, the better the details of the image can be protected. But the ability to filter out noise is limited; on the other hand, the larger the filter window, the better the noise suppression ability can be improved, but the ability to protect the details will be weakened. This kind of contradiction is especially obvious when the noise interference is large in the image. According to the experience: when the impulse noise intensity is greater than 0.2 , the effect of the conventional median filter is not satisfactory. Therefore, it is far from enough to apply the conventional median filter to image denoising, which needs to find a new improved algorithm to solve this contradiction. The filter method of adaptive median filter is the same as that of conventional median filter. They all use a rectangular region of window Sxy, and the difference is in the filtering process. The adaptive filter will change the size of the filter window according to a certain set of conditions[8]. At the same time, when the pixels in the center of the filter window are noise, the value is replaced by the median value, otherwise the current pixel value is not changed. In this way, the output of the filter is replaced by the value of the pixel $(x, y)$ (i.e. the coordinates of the center of the filter window). The optimized median filter algorithm can deal with impulsive noise with greater noise probability and better preserve the details of the image.

\subsubsection{Study on temperature field segmentation based on infrared spectrum isotherm}

Infrared image features are the gradient of temperature field, the shape of the isotherm and the temperature grade. They are closely related to the types of thermal faults and the location of faults in power equipment. Study on the shape parameters of the temperature field and the extraction area are extracted by the image processing method. The purpose of infrared image feature information extraction is to match the image, and then find the similarity between the thermal image and the previous fault shape by image matching. The purpose of the segmentation of the image temperature field is to establish the infrared image sample database of the thermal fault of the power equipment.

The infrared spectrum has rich colors gradually changing according to law, and the area of gradual change color is the main mark of dividing isotherm. In order to identify the isotherms and complete the segmentation of the temperature field, it is necessary to first process the infrared image to gray level, and then to describe the morphological characteristics of the temperature field more clearly. Then, the closed operation of morphology is carried out, and the isotherm is highlighted according to the need. Finally, the connected domain is calculated, and the similar temperature field is segmented and marked. The principle of the algorithm used in the process can be described as follows:

a. Gray level processing of image

Since the photos are all color images, the RGB color pattern is adopted. When dealing with images, three components of RGB should be processed separately. In fact, RGB does not reflect the morphological features of the image, but only the color is deployed from the optical principle. There are many other color model, such as the HSI model. HSI represents color from three components, including the color, saturation and brightness. HSI is more in line with human visual characteristics than RGB.

The color of each pixel in the color image is determined by three components of R, G and B, and each component has a median value of 255 . Such a pixel can have a range of about $16000000(255 * 255 * 255)$ colors. The gray image is a special color image with the same components of $\mathrm{R}, \mathrm{G}$ and $\mathrm{B}$, among which one pixel varies from 255 to three. So, in the digital image processing, the image of various formats is transformed into gray image, so that the amount of the subsequent image is reduced. The description of grayscale images, like color images, still reflects the distribution and characteristics of the overall and local chromaticity and brightness levels of the whole image.

The method is based on the color space of YUV, and the physical meaning of the component of $\mathrm{Y}$ is the brightness of the point. The brightness level is reflected by this value. According to the change relation of RGB and YUV color space, the correspondence of luminance of three color components of $\mathrm{Y}$ and $\mathrm{R}, \mathrm{G}, \mathrm{B}$ can be established: $\quad \mathrm{Y}=0.3 \mathrm{R}+0.59 \mathrm{G}+0.11 \mathrm{~B}$, which is the brightness value and is used to express the gray value of the image.

b. Morphological closed operation

Morphological closed operations are used to eliminate small objects, separate objects at fine points, smooth the boundaries of large objects, and do not change their area at the same time.

Morphological corrosion operation is a process of eliminating boundary points and shrinking boundaries. It can be used to eliminate small and meaningless objects.

Corrosion algorithm: 
Each pixel of the image is scanned with the structure elements of $3 \times 3$; you need to do "and" operations with the structure element and its two valued image; if both are 1, the result image is 1 . pixels; otherwise, it will be 0 . The two valued image can be reduced by one circle.

Expansion is the process of merging all the background points into the object and expanding the boundary to the outside. It can be used to fill holes in objects.

Expansion algorithm:

Each pixel of the image is scanned with the structure elements of $3 \times 3$; you need to do "and" operations with the structure element and its two valued image; if both are 0 , the pixel of the result image is 0 ; otherwise, it will be1; the two valued image can be enlarged in one circle.

First, the image is expanded and then the etching algorithm is used to complete the morphological closed operation.

c. Connected domain calculation

The so-called connected domain is a set of pixels, and the pixels in the set have the following characteristics: First, the gray levels of all pixels are less than or equal to the level of connected domain; second, the pixels in the same connected domain are connected, and there is a path which is completely composed of elements of the set between any two pixels.

Connected domain labeling refers to the identification of pixels that conform to some connected rule to the same target. A suitable data structure is designed to preserve the labeling of each object belonging to each pixel, and to preserve the attributes of the relevant target.

\subsubsection{Study on infrared spectrum feature extraction technology based on temperature field}

We can obtain the temperature field data of different temperature through the infrared image gray processing, the morphological closed operation and connected domain calculation. The temperature field data are identified on the original image and the feature extraction is carried out to obtain the temperature field characteristic parameters of the infrared spectrum[9].

There is a small area in the infrared spectrum of the general fault, which is the highest temperature region. From this region, the temperature radiates to the periphery to form a temperature field with temperature grade. And the highest temperature is a point or a small area, and this point is called the highest temperature point or defect center point. Infrared thermal imager is used to express the temperature of color. The darker the color, the lower the temperature, and the whiter the color is, the higher the temperature is:

$$
I_{\text {def }}=\max \quad\{\mathrm{I}(\mathrm{i}, \mathrm{j})>k(\text { ave }+k s t d)\}
$$

After the original image is gray, the color thermal image is transformed into gray image. In infrared fault diagnosis, it is generally to use defect center to determine the position. According to the characteristics of infrared image, the defect center often corresponds to the pixels of the image contrast of the highest or lowest point. After the infrared image is gray, the defect center is the region or the point with the largest gray value, so the search formula can be expressed as:

$$
I_{\text {def }}=\max \quad\{\mathrm{I}(\mathrm{i}, \mathrm{j})>k(\text { ave }+k s t d)\}
$$

\subsubsection{Research on Fault Diagnosis Technology of infrared spectrum using equipment identification contrast}

For the processing of the algorithm, it is easy to find the coordinates of the highest temperature region points, and then the original image is calibrated and extracted feature processing, so as to get the infrared ray fault center point identification. We can identify the type of equipment and the related parts of the abnormal area of the equipment through identification of equipment by visible spectrum. By contrasting with the infrared spectrum temperature anomaly area, it is found that the temperature anomaly exists in the component of the equipment. Then according to the infrared fault sample library, we can judge, so as to realize the infrared anomaly identification of power equipment.

Therefore, it is necessary to establish an infrared fault sample library. The infrared fault sample library needs to manually classify the infrared spectrum of the equipment according to the type of equipment, and find the typical visible spectrum, normal infrared spectrum and abnormal infrared spectrum of the main equipment. And according to the different parts of the abnormal situation, we make classification. Then, the typical samples are analyzed, the equipment and equipment components are identified, the infrared spectrum temperature field is divided, and the abnormal temperature region is identified. We then linked the visible information and infrared spectrum identification information to record the characteristics of typical faults, and formed a fault sample library.

\section{Summary}

Using infrared spectrum for equipment fault diagnosis, the infrared spectrum should be preprocessed firstly, then the temperature field segmentation based on isotherm is carried out to determine the defect center position of infrared spectrum. Further, the visible image is identified and matched, so that the fault diagnosis is carried out.

\section{References}

1. Chen Heng, 1998. Status and Development of Infrared Diagnostic Technology in China, Laser and Infrared. 5, pp. 378-394.

2. Cheng Yulan, 2002. Practical Technology of Infrared Diagnosis Field, Beijing: Mechanical Industry Press. 
3. Hu Hongguang, 2006. Operation and Management of Power Infrared Diagnosis Technology, Beijing: China Electric Power Press.

4. Wei Feihu, 1990. Pattern Identification and Image Processing. Shanghai: Shanghai Jiao Tong University Press.

5. Shen Yunxia, Zhao Yanli, Zhang Ji, Shen Tao, Wang Yuanzhong, Zhang Jinyu. Application of Infrared Spectroscopy in quality study of traditional Chinese Medicine [J]. World Science and Technology Modernization of traditional Chinese Medicine, 2015, 17 (03): 664-669.

6. Luo Xiyun, Du Yiping, Shen Meihua, Zhang Wenqing, Zhou Xinguang, Fang Shuying, Zhang Xuan. Application of Infrared Spectroscopy in
Identification of fibrous Cultural relics [J]. Spectroscopy and Spectral Analysis, 2015, 35 (01): 60-64.

7. Huang Hua, Zhu Shiping, Liu Bizhen. Architecture and implementation of NIR cloud computing analysis system [J]. Journal of Agricultural Machinery, 2014, (08): 294-298.

8. Yuan Tianjun, Wang Jiajun. Application of near Infrared Spectroscopy and its related Standards [J]. China Agronomy Bulletin, 2013, 29 (20): 190-196.

9. Shao Xueguang, Ning Yu, Liu Fengxia, Li Jihui, Cai Wensheng. Application of Near-infrared Spectroscopy in Inorganic Microanalysis [J]. Journal of Chemistry, 2012, 70 (20): 2109-2114. 